\title{
Skin cancer in regional, rural and remote Australia; opportunities for service improvement through technological advances and interdisciplinary care
}

\section{AUTHORS}

PAMELA ADELSON BSc(Nur) MPH ${ }^{1}$

MARION ECKERT MPH DN MNurSc ${ }^{1}$
1 Rosemary Bryant AO Research Centre, Division of Health Sciences, University of South Australia, Adelaide SA Australia.

\section{CORRESPONDING AUTHOR}

PAMELA ADELSON Rosemary Bryant AO Research Centre, Division of Health Sciences, University of South Australia. 5000. Phone: + 61883021475

Email: Pam.Adelson@unisa.edu.au

\section{ABSTRACT}

Age standardised skin cancers (melanoma and non-melanoma) continue to increase in Australia, although they are stabilising for those under age 40. People living in regional, rural and remote Australia have higher rates of skin cancer and challenges accessing care. Better targeting of skin protection measures and improved opportunistic screening have been promoted as ways to improve care for these populations as have increased use of information technology and upskilling of primary healthcare nurses. The Australian Government supports that Optimal Cancer Care Pathways for skin cancers be used as a key resource in exploring best practice models of care for skin cancer patients both for the development of digital platforms and face to face multidisciplinary teams (MDTs). Better use of technology has been a core recommendation of national health and skin cancer organisations for improving prevention and early detection of skin cancer. Skin cancers, as a primarily visual diagnosis are considered one of the prime areas for technological health interventions. The harnessing of artificial intelligence (AI) technology as a tool for early detection and disease management of skin cancers has great potential to reduce the burden of healthcare costs to the regional, rural and remote community and improve health outcomes.

Keywords: Skin cancer, rural, Australia, nursing, interdisciplinary, artificial intelligence

What is already known about the topic?

- People living in regional, rural and remote Australia have higher rates of skin cancer and challenges accessing care.

- Primary care practitioners - whether nurses or GPs in regional, rural and remote Australia are often the primary contact for skin queries or concerns.

What this paper adds:

- Skin cancers, as a primarily visual diagnosis are considered one of the prime areas for technological health interventions

- Nurses in regional, rural and remote Australia are well positioned to be at the forefront of delivering these emerging technologies 


\section{INTRODUCTION}

\section{SKIN CANCER IN REGIONAL, RURAL AND REMOTE AUSTRALIA}

Skin cancers (melanoma and non-melanoma) are a major public health issue in Australia with two out of three Australians being diagnosed with skin cancer by the age of 70. ${ }^{1}$ The age-standardised incidence rate of melanoma has steadily increased since the 1980 s and estimates of non-melanoma skin cancer (keratinocyte cancers, KCs) are extremely high when compared with overseas rates. ${ }^{2}$ Skin cancer burden is higher among people in regional, rural and remote Australia. ${ }^{3}$ Those outside of major metropolitan cities have the highest rates of melanoma skin cancer, while areas of remote Australia, have lower rates, most likely due to lower rates of skin cancer experienced in Aboriginal populations. ${ }^{2}$ Geographical disparities have also been recently noted in KC with the highest rates in South Australia in agricultural and coastal areas. ${ }^{4}$ This is not altogether surprising given that farmers and outdoor workers have anywhere from three to eight times higher ultraviolet (UV) exposure than indoor workers and sun-protection practices in these populations are noted to be sub-optimal with many reluctant to seek health advice, often waiting until they have a serious health concern. ${ }^{5}$ The most common cited barriers to seeking help for skin cancer detection in rural Australia include; minimising the problem, a desire to be in control, reluctance to show emotion or complain, stoicism and concerns about privacy and waiting times to see a doctor. ${ }^{6}$

\section{PREVENTION, EARLY DETECTION AND SCREENING}

Skin cancer prevention activities for over 40 years in Australia have been credited with the recent stabilisation of melanoma and $\mathrm{KC}$ rates in younger populations $<40$ years of age. Prevention campaigns have evolved by agencies at both the state/territory and national level from whole of population SunSmart campaigns, to targeted campaigns such as those aimed at farmers; Protect your farm's most important asset. You. ${ }^{7}$ Currently skin cancer prevention is primarily funded by State/Territory Governments and organisations, the last nationally funded campaign being from 2006-2010. Cancer Council Australia and Clinical Oncology Society of Australia (COSA) have recommended that the Australian Government fund and conduct an ongoing national mass media social marketing campaign to raise awareness of skin cancer risk and sun protection. ${ }^{8}$

A number of resources for skin cancer prevention and early detection tools have been developed for use by the general public including tools and apps such as those developed by SunSmart, ${ }^{9}$ risk predictor online tools such as the Melanoma Risk Predictor, ${ }^{10}$ Scan Your Skin ${ }^{11}$ and daily ultraviolet index alerts by the Bureau of Meteorology. ${ }^{12}$ However these SunSmart messages may fall short in regional and rural Australia. ${ }^{5}$ In a review of skin protection and public health campaigns for agricultural workers, it has been suggested that increased use of information technology, promoting prevention and early detection at agricultural events and upskilling primary healthcare nurses and other healthcare workers could play an important role in prevention and early detection. ${ }^{5}$ Moving in this direction, a new 2019 Commonwealth funded study, Improving Melanoma and Skin Cancer Awareness in Regional and Rural Australia, coordinated by Melanoma and Skin Cancer (MASC) Trials in collaboration with clinician and patient groups around Australia aims to guide the development and requirements for resources to support people in these areas. ${ }^{13}$

Currently in Australia, population-based screening for skin cancer is not performed due to a lack of evidence either for or against population-based screening. ${ }^{14}$ However, opportunistic screening is common. The Royal Australian College of General Practitioners guidelines currently recommend opportunistic skin checks for people at moderately elevated risk, and skin checks every six to 12 months plus advice on skin self-examination for those at high risk. ${ }^{15}$ Primary care practitioners- whether GPs or nurses in regional, rural and remote Australia are often the primary contact for skin queries or concerns. Skin complaints comprise approximately $15 \%$ of GP consultations, with about one-third of these involving the diagnosis and treatment of a benign or malignant neoplasm. ${ }^{16}$ However, for regional, rural and remote practitioners who may already be stretched to capacity, competing healthcare needs may limit opportunistic skin checks when consideration is given to the time spent on skin examination, visual diagnosis and possible referral to a dermatologist. As $92 \%$ of dermatologists in Australia live and work in major metropolitan cities, ${ }^{17}$ specialist visits are not easy for regional, rural and remote people, although advances in teledermatology has improved access.

Early detection of skin cancers, especially in the case of melanoma is crucial with survival high if acted upon early and localised. Melanoma rates in Australia have decreased by more than $30 \%$ from 2002-2016 for people aged less than 40 which suggest that 40 years of skin cancer prevention programs are having an effect., ${ }^{2,18}$ Melanoma if caught early has a five year survival of $91 \%$, yet in 2019 it is estimated that it will be the ninth most common cause of death from cancer. ${ }^{19}$ Early diagnosis and treatment for all skin cancers decreases the need for invasive and potentially disfiguring surgery especially of the head and neck. It is believed that for older men in particular, the higher proportion of face, scalp and neck tumours could be related to occupational and lifelong sun exposure rather than intermittent extreme exposures. ${ }^{20}$ While KCs mostly do not present a serious disease they are the second most costly cancer (after colorectal) and reflect a disproportionately high burden on the healthcare system. ${ }^{21}$

For some patients, skin cancer will require ongoing management. In the case of $\mathrm{KC}$, due to the multiplicity of 
the disease, the incidence is eight times as high in patients who have had a prior history as for those who have not. ${ }^{22}$ In South Australia, 40\% of people treated for a KC will have another one treated within five years. ${ }^{4}$ For regional, rural and remote practitioners, post-diagnosis skin cancer follow-ups present challenges with the following reasons being cited: (i) workload and time constraints for consultations (ii) GP support, resources and infrastructure (including access to dermatologists for the patient) and (iii) patient factors such as poor health literacy and motivation to attend follow-up. ${ }^{23}$

\section{NATIONAL INQUIRY INTO SKIN CANCER}

Public health recommendations for optimal approaches to the prevention, early detection and management of skin cancer have taken the form of national reports from government and non-government cancer control organisations such as the Department of Health, Australian Institute of Health and Welfare, Cancer Australia, Cancer Council Australia, and professional organisations such as Clinical Oncology Society of Australia. In 2015 The Standing Committee on Health made an Inquiry into Skin Cancer with 63 submissions received from government, non-government, professional organisations, consumers and private individuals. ${ }^{8}$ Amongst the submissions received, a number addressed issues specific to regional, rural and remote health and noted the contribution of nurses and primary healthcare teams in the provision of prevention, opportunistic screening and early diagnosis of skin cancer and called for greater professional support and recognition of their services. These included submissions from the National Rural Health Alliance and the Royal Flying Doctor Service: ${ }^{8}$

Submission 9 exert, the National Rural Health Alliance: Notwithstanding their serious mal-distribution, it remains the case that nurses, doctors and allied health professionals are at the heart of primary care, including for skin cancer. Because nurses are the largest and best-distributed health professionals in rural and remote areas, the best way to provide a workforce for skin cancer care in those areas is through continuing support for their recruitment, retention and professional support. Given the constraints on their time and energies in the vast areas they serve, GPs cannot provide cancer care alone. These local teams also have a key role to play in raising awareness about skin care. Providing more support for these health professionals is imperative to enable them to more effectively undertake the jobs for which they are qualified.

Submission 34 exert, the Royal Flying Doctor Service (RFDS): Skin cancer is a significant issue in rural and remote Australia, with high incidence rates due to a range of higher risk factors. There is also a persistent lack of services available in rural and remote areas. Difficulties in accessing appropriate local services often results in delayed detection and diagnosis and late treatment and requires patients to travel great distances for treatment services. The RFDS strongly recommends: Efforts to improve early diagnosis, treatment and management must take into account the significant lack of dermatology and other specialist services in rural and remote Australia. Recognise GPs and primary healthcare teams as the critical service providers in these areas. There should be a focus on enhancing and ensuring the knowledge and expertise of existing rural and remote service providers. Innovative service models, such as fly-in fly-out and telehealth services, should be considered to provide more dermatology and specialist services to rural and remote communities, in partnership with relevant rural health providers. Specific rural and remote community awareness-raising activities are needed that are appropriate, and targeted to rural populations, taking into account their unique lifestyle factors.

The report from this Inquiry, Skin Cancer in Australia: Our National Cancer, was published in 2017 with 12 recommendations made by the Committee to improve the prevention and treatment of skin cancers in Australia. ${ }^{24}$ Three recommendations are of particular relevance to nurses and primary healthcare workers working in regional, rural and remote Australia. These are:

- Recommendation 8 (part 2): Proficiency in the use of the dermatoscope be included in the practical component of all undergraduate medical courses and in rural nursing training courses.

- Recommendation 11: establish a virtual platform for the multidisciplinary treatment of skin cancer for patients located in regional and remote Australia; and

- further develop and implement best practice models for multidisciplinary care for the treatment of skin cancer patients.

- Recommendation 12: The Committee recommends that the Australian Government ensure that adequate funds are provided for the non-medical support services of skin cancer patients and their families, particularly support services for those rural patients who have to travel for treatment.

\section{IMPROVING OPPORTUNISTIC SCREENING AND EARLY DETECTION THROUGH TECHNOLOGY AND NURSE-LED COLLABORATIVE ARRANGEMENTS IN REGIONAL, RURAL AND REMOTE COMMUNITIES}

Currently the most prevalent tool for identifying skin cancers in general practice is by dermatoscopy. However this requires specialised training and when used by less experienced clinicians, the accuracy can be no better than visual inspection alone. ${ }^{25}$ In 2004, in an effort to improve dermatological services to regional, rural and remote areas underserved by dermatologists, the Australian College of Rural and Remote Medicine introduced the Tele-Derm initiative, initially in Queensland and later expanding nationally. This free online consultation service requires the primary doctor to submit a clear resolution digital photograph to a dermatologist to provide advice. However barriers to doctors using the service have been cited as; lack of remuneration (not billable in the MBS), increasing their workload and too time consuming. ${ }^{26}$ 
The importance and role of multidisciplinary team (MDT) care in skin cancer is recognised as the best practice approach to providing evidence-based cancer care and is supported by the Council of Australian Governments (COAG) as reflected in the Optimal Care Pathways (OCP) for Melanoma and nonMelanoma skin cancers. ${ }^{27}$ Nurses are key MDT members and recognised primary care providers in rural communities, having a broad scope of practice and working collaboratively in advanced and extended roles. ${ }^{28}$ Within their scope of practice nurses have been engaged in preventative and nurseled collaborative arrangements from skin cancer prevention through to early detection and management. Examples of nurse-led skin cancer assessment services in regional, rural and remote areas include initiatives by the Silver Chain organisation in Western Australia, the Skin Cancer Remote Service (SCARS) which provides nurse-led full body skin cancer screening with dermatoscopy and community education and upskilling of nurses. ${ }^{29}$ Nurses have received training in dermatoscopy through training programs such as those offered through the National Professional Development Framework for Cancer Nursing (EdCaN). These learning resources can benefit health professionals not just specialising in cancer and include learning activities for individual nurses and nurse educators, including those for melanoma and current prevention and early detection campaigns. http:|/edcan.org.au/edcan-learning-resources/ using-edcan-resources

Better use of technology has been a core recommendation of national health and skin cancer organisations for improving prevention and early detection of skin cancer. The Australasian College of Dermatologists actively promotes the adoption of telehealth in dermatology services to geographically diverse populations, noting the great potential for telehealth using smart devices for health prevention, education and rapid access to specialist opinion. Currently, practice guidelines for teledermatology are being developed collaboratively by the University of Queensland and the Australasian College of Dermatologists. ${ }^{30}$

Advances in dermatological imaging and improved communication systems will go a long way towards improving diagnostic accuracy and facilitating services to vast geographically diverse areas. New technologies such as surveillance photography, teledermatology, artificial intelligence, and apps to support patient self-examination and triage of clinical examinations also have the potential to reduce overtreatment or unnecessary treatment of some lesions detected in screening. These technologies have great potential to improve equity of access to dermatological services for people living in regional, rural and remote areas. ${ }^{31}$ An emerging technology that is predicted to revolutionise these existing technologies is through artificial intelligence.

\section{EMERGING TECHNOLOGIES: ARTIFICIAL INTELLIGENCE (AI)}

Skin cancers, as a primarily visual diagnosis are considered one of the prime areas for technological health interventions. While there are existing smartphone apps for skin cancer detection, there is little evidence of clinical validation and poorly designed, inaccurate and/or misleading consumer applications may cause harm to patients and risk public safety..$^{32,33}$

In 2017, in a letter to the prestigious science journal Nature, Esteva et al. described how artificial intelligence technology called convolutional neural networks (CNNs) matched or outperformed 21 board-certified dermatologist across three critical diagnostic tasks: keratinocyte carcinoma classification, melanoma classification and melanoma classification using dermatoscopy. ${ }^{34}$ Following the publication of these findings, the international press was alight with headlines such as 'Man against machine, AI is better than dermatologist at diagnosing skin cancer'. It was acknowledged that a dermatologist's clinical diagnosis is based on more than visual and dermatoscopic inspection of a lesion in isolation, however the ability to classify skin lesion images with the accuracy of a specialist has the potential to greatly expand access to skin cancer care. ${ }^{34}$

In a recent trial in dermatology clinics in seven UK hospitals, the study demonstrated that an AI algorithm using different camera types could detect melanoma with a similar accuracy as specialists. The authors concluded that the development of low-cost screening methods, such as artificial intelligencebased services, could transform patient diagnosis pathways, enabling greater efficiencies throughout the healthcare service. ${ }^{2}$ Other specialities that will benefit from AI include radiology and pathology, with predictions that machine learning will soon displace most of this work. ${ }^{35}$ At Memorial Sloan Kettering Cancer Centre in New York, AI is currently being used and further refined by cancer pathologists with results showing that machine-learning is approaching 100\% sensitivity for biopsies for prostate, skin and breast cancer. ${ }^{36}$

There are still issues to overcome in AI technology with regards to early detection of skin cancers including different skin backgrounds (most have been tested on caucasian populations), varying camera angles and lighting. It has been recommended in a systematic review and in several other studies assessing CNN that to improve the robustness of the CNN classifier, establishment of an open-access, standardised, large skin tumour image dataset, which includes both rare tumours/subtypes and all ethnicities, is mandatory. ${ }^{37,38}$ In Australia, the University of Queensland is part of the International Skin Imaging Collaboration (ISIC) Melanoma Project which is addressing issues concerning standards and technology and developing an open source public access archive of skin images. ${ }^{39}$ 
While an accurate history and assessment will always be as essential component of diagnosis and management, initial visual inspection and classification by AI will go a long way towards early detection in communities where access to GPs and specialist doctors are difficult such as in regional, rural and remote Australia. Smartphone applications do not currently incorporate AI technology; however, it is just a matter of time before the technology is readily available for GPs and nurses to make the best use of this technology through smartphones. In a thoughtful editorial regarding AI and melanoma by Associate Professor Mar of Monash University and Professor Soyer of Queensland University, it was noted that AI promises a more standardised level of diagnostic accuracy, such that all people, regardless of where they live or which doctor they see, will be able to access reliable diagnostic assessment. ${ }^{40}$

\section{WHERE TO FROM HERE?}

The harnessing of AI technology as a public health tool for early detection and disease management of melanoma and $\mathrm{KC}$ will differ in approach but has great potential to reduce the burden of healthcare costs to the community and improve health outcomes. The Australian Government supports that Optimal Cancer Care Pathways be used as a key resource in exploring best practice models of care for skin cancer patients both for the development of digital platforms and face to face MDTs. ${ }^{27}$ Delivering healthcare to regional, rural and remote communities is challenging and nurses are increasingly called upon to work to the full scope of practice as well as working in advanced practice roles in Australian rural health services in response to workforce demands. ${ }^{41}$ Nurses as primary healthcare providers in regional, rural and remote Australia are well positioned to be at the forefront of delivering these emerging technologies, whether working in advanced nursing practice roles and/or working collaboratively with GPs and dermatologists through established multidisciplinary organisations that focus on delivering services in regional, rural and remote areas.

As skin cancers are initially assessed on visual inspection and prompt action is likely to result in cure, opportunistic screening in high risk populations with AI has great promise by means of improving diagnostic accuracy and decreasing the high proportion of investigations of suspicious benign lesions. It has been noted that despite the benefits of telehealth technologies, the actual uptake and integration into mainstream practice has been slow and fragmented, with many pilot programs not migrating into sustained services..$^{42}$ The introduction of AI potentially could remove some of these telehealth barriers, such as the need for real-time consultations and administrative burden. In a systematic review of telehealth services in rural Australia, six factors were identified that influenced success and sustainability of telehealth services. These were: vision, ownership, adaptability, economics, efficiency and equipment. ${ }^{42}$
Translation into a real-world scenario with AI technology is not too distant in the future. Mobile app technology and repositories of public source images of different skin lesions are becoming available. AI for skin cancer early detection is a good technological alternative for which there is good evidence, and is likely to be well received, efficacious and cost effective. There is likely to be minimal training involved and with better support and utilisation of the existing primary care workforce the technology should be readily adaptable within existing services. Careful planning and collaboration between health services will be needed to ensure that best practice is applied in bringing these services to those in regional, rural and remote Australia. As a needed service that will be low cost, efficacious and has great potential for cost savings to the health system, the time is ripe for exploring opportunities to bring this emerging technology to those who will most benefit from it.

Funding support: No funding was received for this manuscript.

Declaration of conflicting interests: The authors have no conflicts of interest to declare.

\section{REFERENCES}

1. Cancer Council Australia. Skin Cancer. 2019; https://www. cancer.org.au/about-cancer/types-of-cancer/skin-cancer.html Accessed 11/11/2019.

2. Australian Institute of Health and Welfare. Skin cancer in Australia. Canberra: AlHW;2016

3. Australia Institute of Health and Welfare. Rural, regional and remote health: indicators of the health status and determinants of health. In. Canberra: AlHW; 2008.

4. Adelson P, Sharplin GR, Roder DM, Eckert M. Keratinocyte cancers in South Australia: incidence, geographical variability and service trends. Aust Nz J Publ Heal. 2018;42(4):329-33.

5. Smit-Kroner C, Brumby S. Farmers sun exposure, skin protection and public health campaigns: An Australian perspective. Prev Med Rep. 2015;2:602-607.

6. Fennell KM, Martin K, Wilson CJ, Trenerry C, Sharplin G, Dollman J. Barriers to Seeking Help for Skin Cancer Detection in Rural Australia. J Clin Med. 2017;6(2):19.

7. Cancer Council Victoria Vic Health. Protect your farm's most important assest. You. 2012; https://www.sunsmart.com.au/ downloads/resources/brochures/farmers-brochure.pdf

8. Parliament of Australia. Inquiry into Melanoma and nonmelanoma skin cancers. Submissions received by the Committee. 2014; https://www.aph.gov.au/Parliamentary_ Business/Committees/House/Health/Skin_Cancer/Submissions

9. SunSmart smartphone app. http://www.sunsmart.com.au/ resources/sunsmart-app

10. QIMR Berghofer Medical Research Institute. Melanoma Risk Predictor. https://publications.qimrberghofer.edu.au/Custom/ QSkinMelanomaRisk

11. Skin Cancer College Australasia. Scan Your Skin https://www. scanyourskin.org/risk-prediction-tool/

12. Australian Government Bureau of Meterology. About UV and sun protection times. http://www.bom.gov.au/uv/ 
13. Centre for Health Economics Research and Evaluation. CREST Update Sept 2019. Melanoma and Skin Cancer Trials (MASC Trials). 2019; https://www.uts.edu.au/sites/default/ files/2019-10/crest-newsletter-2019-09.pdf Accessed $11 / 11 / 2019$.

14. Australian Government Standing Committee on Screening. http://www.cancerscreening.gov.au/internet/screening/ publishing.nsf/Content/standing-committee-on-screening

15. Royal Australian College of General Practitioners. Skin checks. https://www.racgp.org.au/afp/2012/july/skin-checks/ Accessed 8/11/2019, 2019

16. Harvey NT, Chan J, Wood BA. Skin biopsy in the diagnosis of neoplastic skin disease. Aust Fam Physician. 2017;46(5):289-94.

17. Department of Health. Australia's Future Health WorkforceDermatology. In. Australia 2017.

18. Montague M, Borland R, Sinclair C. Slip! Slop! Slap! and SunSmart, 1980-2000: Skin cancer control and 20 years of population-based campaigning. Health Educ Behav. 2001;28(3):290-305.

19. Australian Government. Cancer Australia. Melanoma of the skin. https://melanoma.canceraustralia.gov.au/ Accessed 11/11/2019.

20. Curchin DJ, Harris VR, McCormack CJ, Smith SD. Changing trends in the incidence of invasive melanoma in Victoria, 1985-2015. MJA. 2018;208(6):265-9.

21. Australian Institute of Health and Welfare. Health System Expenditure on Cancer and Other Neoplasms in Australia: 2008-09. In. Canberra (AUST): AlHW; 2013.

22. Pandeya N, Olsen CM, Whiteman DC. The incidence and multiplicity rates of keratinocyte cancers in Australia. MJA. 2017;207(8):339-43.

23. Aung E, Campbell D, Mitchell E. Post diagnosis skin cancer follow up in rural general practice. Aust J Gen Pract. 2019;48:222-8.

24. Australian Government. Australian Government reposonse to the House of Representatives Standing Committee on Health Report: Skin Cancer in Australia: Our National Cancer. In. Canberra ACT: Department of Health 2017.

25. Jones OT, Jurascheck LC, van Melle MA, et al. Dermoscopy for melanoma detection and triage in primary care: a systematic review. BMJ Open. 2019;9(8):e027529.

26. Liu R. Evaluating Artificial Intelligence and Telemedicine-based Care Models in Dermatology. Journal of Mobile Technology in Medicine. 2019;8(1):50-2.

27. Cancer Council Australia. Optimal cancer care pathways https://www.cancer.org.au/health-professionals/optimalcancer-care-pathways.html Accessed 20/11/2019

28. CRANAplus. Rural Nursing. 2019; https://crana.org.au/ resources/practice/rural-nursing Accessed 11/11/2019.

29. Christensen S. SCARS- Skin Cancer Assessment Remote Service- Nurse led clinics. Paper presented at: CRANAplus 37th Conference 2019; Lovedale, NSW.

30. The University of Queensland Centre for Online Health Development of practice guidelines for teledermatology in Australia. https://coh.centre.uq.edu.au/development-practiceguidelines-teledermatology-australia Accessed 25/11/2019

31. Weber M, Marshall H, Rankin N, et al. Cancer screening in Australia: future directions in melanoma, Lynch syndrome, and liver, lung and prostate cancers. Public Health Res Pract. 29(2):pii2921910.
32. Phillips M, Marsden $H$, Jaffe W, et al. Assessment of Accuracy of an Artificial Intelligence Algorithm to Detect Melanoma in Images of Skin Lesions. JAMA Network Open. 2019;2(10):e1913436-e1913436.

33. Chuchu N, Takwoingi Y, Dinnes J, et al. Smartphone applications for triaging adults with skin lesions that are suspicious for melanoma. Cochrane Database Syst Rev. 2018; Dec 4(12): CD013192.

34. Esteva A, Kuprel B, Novoa RA, et al. Dermatologist-level classification of skin cancer with deep neural networks. Nature. 2017; $542: 115$

35. Obermeyer Z, Emanuel EJ. Predicting the Future - Big Data, Machine Learning, and Clinical Medicine. N Engl J Med. 2016;375(13):1216-9.

36. Campanella G, Hanna MG, Geneslaw L, et al. Clinical-grade computational pathology using weakly supervised deep learning on whole slide images. Nat Med. 2019;25(8):1301-9.

37. Brinker TJ, Hekler A, Utikal JS, et al. Skin Cancer Classification Using Convolutional Neural Networks: Systematic Review. J Med Internet Res. 2018;20(10):e11936.

38. Fujisawa Y, Inoue S, Nakamura Y. The Possibility of Deep Learning-Based, Computer-Aided Skin Tumor Classifiers. Frontiers in Medicine. 2019;6:191.

39. The University of Queensland Dermatology Research Centre International Skin Imaging Collaboration (ISIC) Melanoma Project. https://dermatology-research.centre.uq.edu.au/ international-skin-imaging-collaboration-isic-melanoma-project Accessed 25/11/2019.

40. Mar VJ, Soyer HP. Artificial intelligence for melanoma diagnosis: how can we deliver on the promise? Annals of Oncology. 2018;29(8):1625-1628.

41. Cant R, Birks M, Porter J, Jacob E, Cooper S. Developing advanced rural nursing practice: A whole new scope of responsibility. Collegian. 2011;18(4):177-182.

42. Bradford NK, Caffery LJ, Smith AC. Telehealth services in rural and remote Australia: a systematic review of models of care and factors influencing success and sustainability. Rural and remote health. 2016;16(4):3808 